\title{
Intraureteral lidocaine for ureteral stent symptoms post-ureteroscopy: A randomized, phase 2, placebo-controlled trial
}

\author{
Gregory Roberts, $M D^{1 *}$; Robert Leslie, $M D^{1 *}$; Sylvia Robb, $M D^{1} ;$;. Robert Siemens, $M D^{1,2}$; Darren Beiko, $M D^{1}$
}

*Co-first author

'Department of Urology; ${ }^{2}$ Department of Oncology; Queen's University, Kingston, ON, Canada

Cite as: Can Urol Assoc J 2017;11 (10):326-30. http://dx.doi.org/10.5489/cuaj.4408

\section{Abstract}

Introduction: Ureteral stent and ureteral manipulation-related pain is a significant complication for patients undergoing ureteroscopy. Herein, we report a phase 2, randomized trial to assess efficacy of direct instillation of intraureteral lidocaine in reducing postoperative pain and ureteral stent symptoms.

Methods: We performed a randomized, double-blinded trial of patients undergoing elective ureteroscopy for ureteral calculi. Patients were randomized to direct instillation of $2 \%$ lidocaine plus bicarbonate, or to normal saline as control. The primary outcome of interest was early postoperative pain scores. Patients completed10-point visual analog pain scale at one-hour, two-hour, four-hour, 24-hours, four- and seven-day time points. Other outcome measurements collected included a medication diary and voiding questionnaire.

Results: A total of 41 patients were randomized in the study. Mean flank pain scores at one hour were $2.2( \pm 2.9)$ vs.1.9 $( \pm 2.4)$ in the intervention and placebo group, respectively $(p=0.84)$. There was no significant difference at any time point between the intervention and placebo groups in patient-reported pain scores. Patients reported lower dysuria scores at all time points in the lidocaine group, however, none reached statistical significance. There was no difference in complication rates or adverse effects between groups. Conclusions: In this randomized, phase 2 study, direct instillation of lidocaine into the ureter did not appear to significantly improve pain or voiding symptoms following stented ureteroscopy.

\section{Introduction}

Ureteroscopy (URS) has evolved to become a highly effective first-line modality for ureteral and renal stones. ${ }^{1-3}$ Following ureteroscopic stone manipulation, ureteral stents are often left indwelling on a short-term basis, typically ranging from days and up to a few weeks. Despite the importance of ureteral stenting for urological indications, ${ }^{4}$ stent-related morbidity is ubiquitous. Ureteral stents are associated with significant morbidity, including urinary tract infection (UTI), hematuria, bothersome lower urinary tract symptoms (LUTS), as well as flank, abdominal, and pelvic pain. These complications have been reported by more than $80 \%$ of patients in studies $^{5}$ and have significant effects on healthcare resources and time off work.

Ureteral stent morbidity has been addressed in recent studies assessing oral medications, ${ }^{6}$ comfort stents, ${ }^{7}$ biodegradable stents, ${ }^{7}$ drug-eluting stents, ${ }^{7,8}$ and novel stent designs. ${ }^{9}$ Unfortunately, despite such advances, ureteral stent-related symptoms remain an unresolved issue.

A novel approach to addressing the problem of stentrelated symptoms has been explored with direct drug delivery into the urinary tract to assess its impact on stent pain and voiding symptoms. ${ }^{8}$ The successful use of intravesical ketorolac has been demonstrated to reduce bladder symptoms with refluxing stents following shock wave lithotripsy; that same study demonstrated safety of passive instillation of lidocaine into the upper urinary tract via intravesical instillation with ureteral stent. ${ }^{8}$

In the interstitial cystitis (IC) and chronic pelvic pain syndrome (CPPS) patient populations, intravesical lidocaine has been proven to be safe and effective. ${ }^{10,11}$ Prior studies have investigated the use of intravesical lidocaine, as well as ureteral orifice injections of lidocaine on pain and LUTS; however, pharmacokinetic studies have shown that lidocaine is not sufficiently absorbed by human bladders, as conversion of the lidocaine to the lipid-soluble base form does not occur in the acidic bladder environment. ${ }^{10,11}$ Previous clinical studies have demonstrated that lidocaine is absorbed and provides safe and effective anesthetic in the bladder if alkalinized to a $\mathrm{pH}$ of $8.0 .{ }^{10,11}$ Consequently, there is a requirement for urinary alkalinization in order to improve lidocaine's urothelial absorption. To date, no study has examined the effect of ureteral instillation of alkalinized lidocaine on pain and LUTS.

This phase 2, randomized study was designed to assess the safety and efficacy of upper tract-instilled alkalinized lidocaine in patients with ureteral stents following URS. Our primary objective was to assess the efficacy of intraureteral 
lidocaine in reducing early postoperative ureteral stent-related pain. Our secondary objectives were to confirm the safety of intraureteral instillation of lidocaine, in addition to assessing the efficacy of intraureteral lidocaine in reducing LUTS.

\section{Methods}

We performed a prospective, double-blinded, randomized clinical trial. Forty-one patients undergoing URS requiring ureteral stent with tether were randomized to one of two groups. Randomization was performed in the operating room by concealed numbered envelopes with the original target sample size of 48 patients. The intervention group received an intraureteral instillation of alkalinized lidocaine and the control group received an intraureteral instillation of normal saline of similar volume. Patients over 18 years of age who were able to consent and were willing to complete the ureteral stent questionnaires were eligible for the trial. The study design was reviewed and approved by the research ethics board of Queen's University. The consort diagram with exclusion criteria is listed in Supplementary Fig. 1.

Patients were included in the study if they were booked for elective URS for ureteral or renal stone disease and were determined by the treating surgeon to likely require shortterm postoperative stenting. Patients were randomized intraoperatively if the case included the placement of a shortterm ureteral stent with tether. At the termination of the URS, and immediately before ureteral stent placement, the bladder was emptied with immediate instillation of study drug into the renal pelvis and proximal ureter via a 5 French open-end ureteral catheter. Two sets of syringes were prepared in the hospital pharmacy, and syringe \#1 was instilled first, immediately followed by syringe $\# 2$. For the treatment group, syringe \#1 contained $10 \mathrm{ml}$ of $2 \%$ lidocaine hydrochloride and syringe \#2 contained $5 \mathrm{ml}$ of $8.4 \%$ sodium bicarbonate. For the control (placebo) group, syringe \#1 contained $10 \mathrm{ml}$ of $0.9 \%$ sodium chloride and syringe \#2 contained $5 \mathrm{ml}$ of $0.9 \%$ sodium chloride. A Double Pigtail ureteral stent by Gyrus Acmi was positioned two minutes following instillation. Stents size ranged from $6 \times 24 \mathrm{~cm}$ to $6 \times 30 \mathrm{~cm}$ based on patient height.

Study patients were assessed as per usual procedure in the post-anesthetic care unit immediately following their procedure. Each patient completed a visual analogue pain scale (VAS) at one-hour, two-hour, four-hour, 24-hour and fourday time points. Prior to discharge, patients were also given a medication diary, a voiding diary, and self-report symptom questionnaires. Opioid, non-steroidal anti-inflammatory drug (NSAID), acetaminophen, and alpha-blocker use were documented for the week following surgery. Perioperative and postoperative analgesia was administered on a usual care protocol. Patients removed their ureteral stents at home by pulling on the tether on postoperative Day 3. Followup occurred in person on postoperative Day $7 \pm 1$ day, at which time the final questionnaire was completed. Additionally, a kidneys-ureters-bladder (KUB) $\mathrm{x}$-ray, urinalysis, and urine culture were performed at the one-week visit, along with documentation of any concomitant medications and adverse events occurring during the course of the study.

\section{Statistical analysis}

For this phase 2 study, the primary outcome measured was early postoperative pain as measured by the mean VAS pain score. Secondary endpoints were urinary frequency, pain mediation diary, and ureteral stent symptoms assessed at different time points during the study. Mann-Whitney tests were used to compare median values between groups. ANOVA was used to compare VAS data. All statistical tests were performed using Sigma Stat. The sample size calculations were based off previous similar studies and alkalinized lidocaine estimated effect size calculations. ${ }^{11,12}$ We hypothesized our mean pain score to be 2.5 on the VAS. Using a power of $80 \%$ and alpha of 0.5 , a sample of 20 patients per group was powered to detect a $30 \%$ difference in VAS.

\section{Results}

Table 1 represents baseline patient, stone, and operative characteristics, and demonstrates that the groups were wellbalanced. Mean patient age was 51 years in both groups. The distribution of stone location was comparable, with 10

\begin{tabular}{|c|c|c|c|c|}
\hline & $\begin{array}{c}\text { Placebo } \\
(n=23)\end{array}$ & $\begin{array}{c}\text { (SD or } \\
\%)\end{array}$ & $\begin{array}{l}\text { Lidocaine } \\
\quad(n=18)\end{array}$ & $\begin{array}{c}\text { (SD or } \\
\%)\end{array}$ \\
\hline Mean age, years & 51 & \pm 15 & 51 & \pm 18 \\
\hline \multicolumn{5}{|l|}{ Sex, $n$} \\
\hline Male & 14 & 0.61 & 9 & 0.5 \\
\hline Female & 9 & 0.39 & 9 & 0.5 \\
\hline \multicolumn{5}{|l|}{ Mean body size } \\
\hline Height, $\mathrm{cm}$ & 168 & \pm 10 & 170 & \pm 11 \\
\hline Weight, kg & 82 & \pm 15 & 91 & \pm 21 \\
\hline \multicolumn{5}{|l|}{ Mean stent size } \\
\hline Length, cm & 26 & \pm 1.2 & 26 & \pm 1.4 \\
\hline \multicolumn{5}{|l|}{ Stone location, $\mathrm{n}$} \\
\hline Left & 14 & 0.61 & 11 & 0.61 \\
\hline Right & 9 & 0.39 & 7 & 0.39 \\
\hline Renal pelvis & 9 & 0.39 & 8 & 0.44 \\
\hline Proximal ureter & 5 & 0.22 & 4 & 0.22 \\
\hline Distal ureter & 8 & 0.39 & 6 & 0.33 \\
\hline Mean stone size, $\mathrm{mm}$ & 7.7 & \pm 3 & 8.8 & \pm 6 \\
\hline $\begin{array}{l}\text { Mean surgical duration, } \\
\text { minutes }\end{array}$ & 60.8 & \pm 19 & 66.7 & \pm 21 \\
\hline
\end{tabular}


ureteric and eight renal stones in the intervention group, compared to 13 ureteric and nine renal stones in the control group. Overall mean stone size was $8.4 \mathrm{~mm}( \pm 4.8)$. The study was terminated after 41 of the target 48 patients were completed, due to logistical and resource constraints. After the allocation code was broken, there were 18 patients in the treatment group and 23 patients in the control group.

Mean postoperative pain scores ranged from 1-3 on a 10-point Likert scale throughout the study period (Figs. 1A, 1B). There was no significant difference in mean pain scores at any of the measured time points. Early postoperative mean flank pain scores were numerically lower at the one-hour and two-hour time points in the control group at $1.89( \pm 2.4)$, and $1.0( \pm 1.5)$ as compared to $2.2( \pm 2.9)$ and $1.6( \pm 2.7)$ in the intervention group ( $p=0.91$ and $p=0.92$, respectively). Mean abdominal pain scores at the one- and two-hour mark were $1.9( \pm 2.5)$ and $1.1( \pm 1.8)$ compared to $1.9( \pm 3.1)$ and $1.4( \pm 2.4)$, in the placebo and lidocaine groups $(p=1.0$ and $\mathrm{p}=0.79$ ), respectively. There was no statistical difference of the VAS scores between groups at any time points. Finally, at no time period was there a predefined two-point difference of pain scores between groups, suggesting that intraureteral alkalized lidocaine did not lead to a meaningful difference in early postoperative pain after URS and stenting after lithotripsy. Similarly, we did not find a significant difference at any time point in frequency, urgency, or dysuria scores between groups (Figs 2A, 2B, 2C). The only marginal trend to benefit came with a mean dysuria value on average 0.7 points lower at each time point in the intervention group $(p=0.09)$.

Postoperative requirements for opioids, NSAIDs, acetaminophen, and alpha- blockers are displayed in Table 2 . There was a trend to increased opioid equivalents in the intervention group, mean $31 \mathrm{mg}$ vs. $12 \mathrm{mg}(p=0.9)$.
Ibuprofen and acetaminophen use were very similar, with means of $189 \mathrm{mg}$ vs. $183 \mathrm{mg}$ and $1179 \mathrm{mg}$ vs. $1038 \mathrm{mg}$ in the intervention and control groups, respectively. Alphablocker use was similar between groups: $55 \%$ of patients on medication in the intervention group vs. $65 \%$ in the control group. There were five adverse events: one ClavienDindo classification I and four Clavien-Dindo classification II. Three of these events were in the control group and two were in the intervention group. None were believed to be secondary to the intervention drug. There were no seizures or signs of lidocaine toxicity reported in either group.

\section{Discussion}

This randomized, phase 2, placebo controlled study assessing the safety and efficacy of postoperative intraureteral alkalized lidocaine did not demonstrate a significant benefit of the patient's pain experience. It was anticipated that any expected minimally detectable change would be most obvious at early time points; however, the mean VAS pain score at one hour was very similar between groups: $2.2( \pm 2.9)$ for the lidocaine group and $1.89( \pm 2.4)$ for placebo. Similarly, there was no significant difference in self-reported pain between groups at any time point in the study followup. Despite the marginal reduction in mean dysuria values at each time point, there was no significant benefit of the intervention with respect to postoperative LUTS in any self-reported symptom score. The relationship of decrease in LUTS reported at Days 4 and 7 in the treatment group are difficult to attribute to lidocaine based on its short duration of action.

These results are similar to previous studies. ${ }^{12,13}$ Sur et $\mathrm{al}^{13}$ randomized 22 patients to preoperative injection of $0.5 \%$ ropuvicaine or normal saline around the ureteric

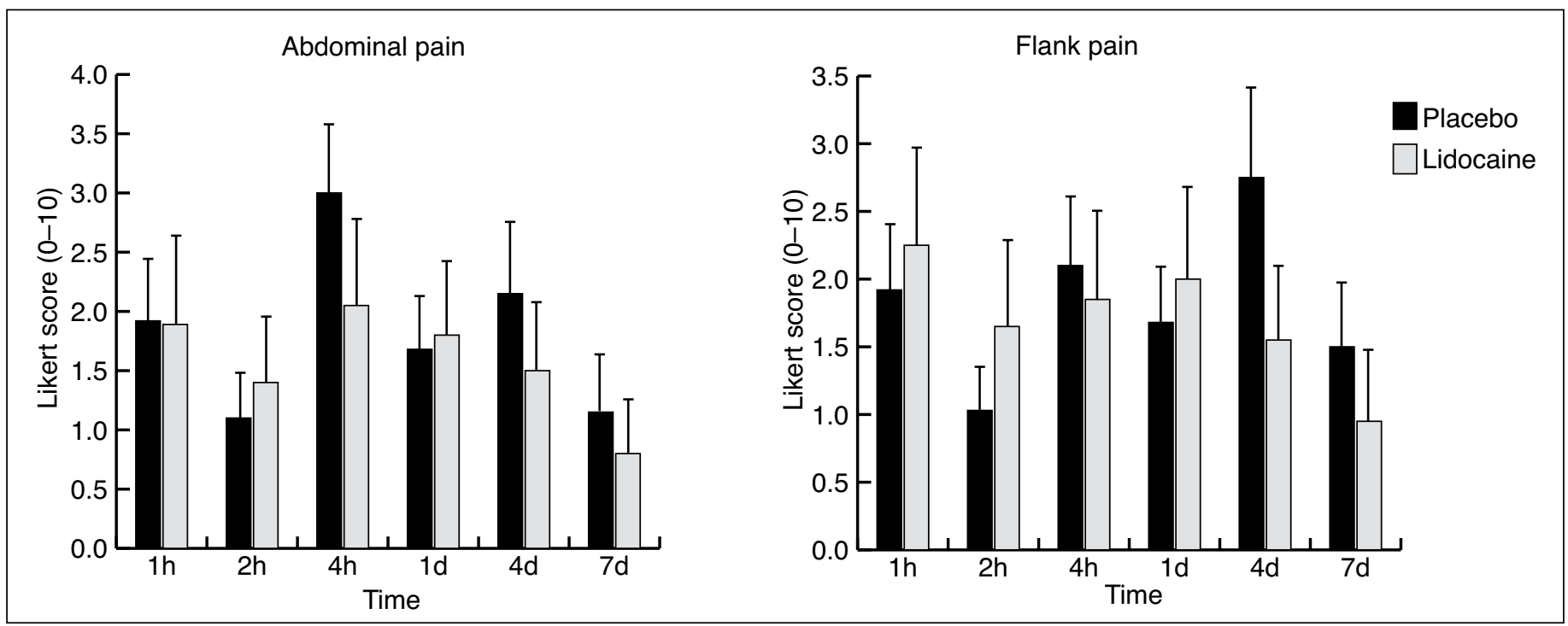

Fig. 1. Patient-reported ( $\boldsymbol{A}$ ) abdominal pain scores; and (B) flank pain scores; each at one-hour, two-hour, four-hour, one-day, four-day, and seven-day time points. There is no significant difference in either abdominal or flank scores between groups at any time point. 


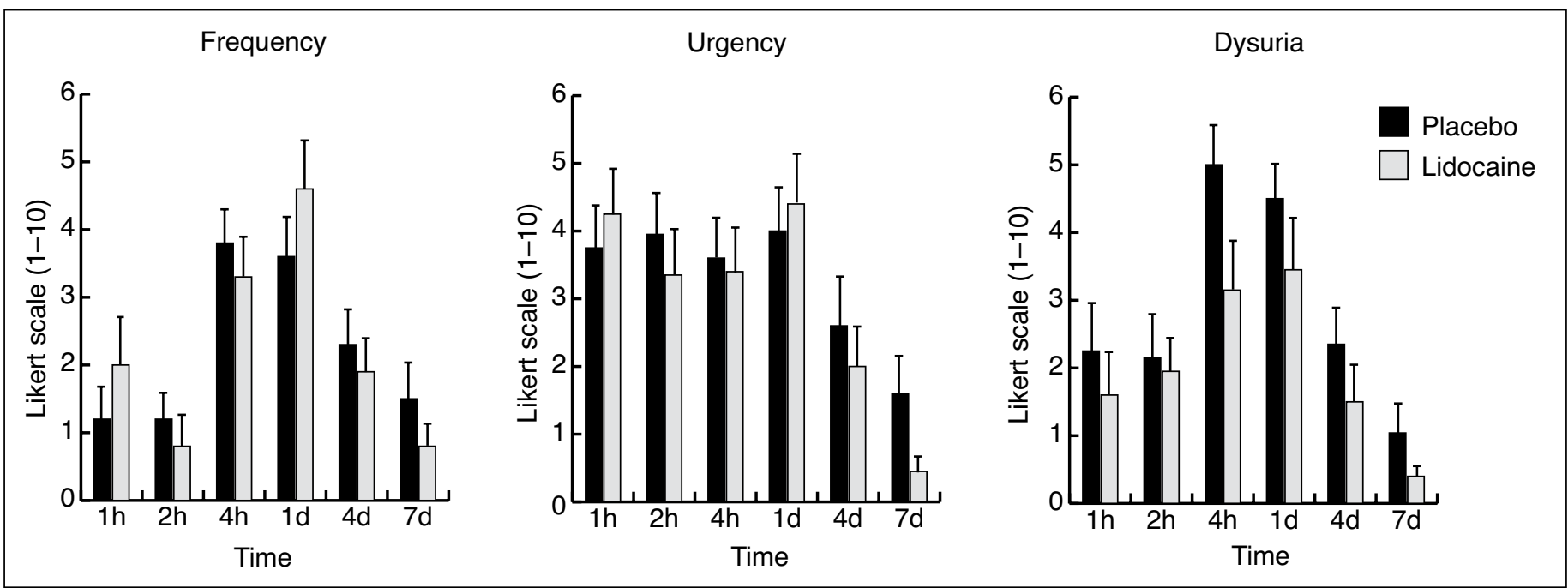

Fig. 2. Patient-reported (A) frequency scores; (B) urgency scores; and (C) dysuria scores; each at each at one-hour, two-hour, four-hour, one-day, four-day, and seven-day time points. There is no significant difference in and voiding scores between groups at any time point. There is a trend for decreased dysuria scores in the lidocaine group $(p=0.09)$.

orifice. They found trends to decreased pain scores up to eight hours, but no significant differences. Beiko et al ${ }^{12}$ randomized 42 patients into four group; three groups received interventions of either intravesical oxybutynin, lidocaine, or ketorolac and used intravesical normal saline as control. They observed significant reduction in pain scores at one hour in the ketorolac and oxybutynin groups, but not in the intravesical lidocaine group. They did not alkalinize the bladder to optimized lidocaine absorption. Based on the work of Henry et $\mathrm{al}^{10}{ }^{10}$ we hypothesized that alkalinizing lidocaine would improve urothelial absorption and lead to improved pain and voiding symptoms; however similar to previous studies, this study demonstrated no improvement of flank or abdominal pain with this modification to lidocaine delivery to the urothelium. We did not observe the significant 4.2-point reduction in mean pain score observed in the previous study in IC patients who received intravesical alkalinized lidocaine. ${ }^{10}$ This may be due to the different etiology of bladder/pelvic pain in the IC patient population.

We did not find a difference in secondary endpoints between groups with respect to postoperative LUTS. PostURS frequency, urgency, or dysuria scores between groups

\begin{tabular}{|c|c|c|c|c|c|}
\hline & \multicolumn{2}{|c|}{ Placebo } & \multicolumn{2}{|c|}{ Lidocaine } & \multirow[t]{2}{*}{$\mathbf{p}$} \\
\hline & Mean & SD & Mean & SD & \\
\hline $\begin{array}{l}\text { Morphine equivalence, } \\
\text { mg }\end{array}$ & 12.2 & \pm 9.4 & 31.1 & \pm 10.1 & 0.09 \\
\hline Ketoralac, mg & 7.6 & \pm 22.0 & 3.2 & \pm 7.1 & 0.28 \\
\hline Ibuprofen, mg & 183.3 & \pm 350.9 & 189.5 & \pm 308.7 & 0.97 \\
\hline \multirow[t]{2}{*}{ Acetaminophen, mg } & 1037.5 & \pm 688.5 & 1178.9 & \pm 674.2 & 0.71 \\
\hline & n (\%) & & n (\%) & $\mathbf{p}$ & \\
\hline Tamsulosin & $15(65)$ & & $10(55)$ & 0.53 & \\
\hline
\end{tabular}

were generally similar at all time points. Although there was some trend to improved frequency, urgency, and dysuria scores after stent removal, these findings were not statistically significant. Sur et $\mathrm{al}^{13}$ also evaluated ropuvicaine submucosal injections around the ureteric orifice and found no significant change in voiding symptoms. This supports the findings of Miyaoka and Monga' $\mathrm{s}^{14}$ literature review, of "prevention as the best treatment" for stent-related morbidity.

Damiano et $\mathrm{al}^{15}$ and Wang et $\mathrm{al}^{8}$ have shown tamsulosin to be an efficacious therapy for stent-related symptoms. Usual care medication administration was used in our study and we found $65 \%$ of patients in the placebo group and $55 \%$ of patients in the lidocaine group were on tamsulosin. One of the limitations of our study was the lack of control for postoperative co-intervention with the use of alpha-blocker and analgesia, as shown in Table 2. We attribute some of the differences in analgesic use to patients' baseline use of medications for comorbid conditions, while other differences may be attributed to provider (anesthesiologist, family physician, urologist) preferences; however, within the confines of this "effectiveness" study after URS, there appears to be no demonstrable benefit of intraureteral alkalized lidocaine. This current pilot study was limited to a relatively small sample size, although it was powered to assess a moderate effect size, based on similar studies, in order to determine the utility of a larger, multicentre, prospective trial. Finally, despite being consistent with much of the published data using pain, frequency, urgency, and dysuria assessments on a 10-point Likert scale, ${ }^{6,12,13}$ our questionnaire was not validated specifically for postoperative ureteral/bladder pain. We used a five-item questionnaire for ease and efficiency of use for this pilot study, as opposed to the 38-item Ureteral Stent Symptom Questionnaire (USSQ). ${ }^{16}$ 


\section{Conclusion}

We performed a double-blinded, randomized, controlled trial comparing intraureteral alkalized lidocaine and placebo for ureteral stent pain and voiding symptoms. Alkalinized lidocaine appears safe for intraureteral administration; however, no clinically significant differences in pain or voiding scores were demonstrated between groups.

Competing interests: Dr. Leslie has received an educational grant from Ferring Canada. The remaining authors report no competing personal or financial interests.

This paper has been peer-reviewed.

\section{References}

1. Dretler SP. Ureteral stone disease. Options for management. Urol Clin North Am 1990; 17:217-30.

2. Park H, Park M, Park T. Two-year experience with ureteral stones: Extracorporeal shockwave lithotripsy vs. ureteroscopic manipulation. J Endourol 1998;12:501-4. https://doi.org/10.1089/end.1998.12.501

3. Segura JW, Preminger GM, Assimos DG, et al. Ureteral stones clinical guidelines panel summary report on the management of ureteral calculi. J Urol 1997;158:1915-21. https://doi.org/10.1016/S0022$5347(01) 64173-9$

4. Saltzman B. Ureteral stents - indications, variations, and complications. Endourology Update1988;15:481-91.

5. Joshi HB, Stainthorpe A, Keeley FX Jr, et al. Indwelling ureteral stents: Evaluation of quality of life to aid outcome analysis. J Endourol 2001;15:151-4. https://doi.org/10.1089/089277901750134421

6. Wang CJ, Huang SW, Chang $\mathrm{CH}$. Effects of tamsulosin on lower urinary tract symptoms due to double-J stent: A prospective study. Urol Int 2009;83:66-9. https://doi.org/10.1159/000224871

7. Krambeck AE, Walsh RS, Denstedt JD, et al for the Lexington Trial Study Group. A novel drug eluting ureteral stent: A prospective, randomized, multicentre clinical trial to evaluate the safety and effectiveness of a ketorolac loaded ureteral stent. J Urol 2010; 183:1037-42. https://doi.org/10.1016/i. juro.2009.11.035
8. Kahokehr A, Sammour T, Vather R, et al. Systemic levels of local anaesthetic after intra-peritoneal application —a systematic review. Anaesth Intensive Care 2010;38:623-38.

9. Lee C, Kuskowski M, Premoli J, et al. Randomized evaluation of ureteral stents using validated symptom questionnaire. J Endourol 2005; 19:990-3.

10. Henry RA, Patterson L, Nickel C, et al. Alkalinized intravesical lidocaine to treat interstitial cystitis: Absorption kinetics in normal and interstitial cystitis bladders. Urology 2001;57:119. https://doi.org/10.1016/ S0090-4295(01)01069-X

11. Henry R, Patterson L, Avery N, et al. Absorption of alkalized intravesical lidocaine in normal and inflamed bladders: A simple method for improving bladder anesthesia. J Urol 2001;165:1900-3. https://doi.org/10.1097/00005392-200106000-00014

12. Beiko DT, Watterson JD, Knudsen BE, et al. Double-blind, randomized, controlled trial assessing the safety and efficacy of intravesical agents for ureteral stent symptoms after extracorporeal shockwave lithotripsy. J Endourol 2004;18:723-30. https://doi.org/10.1089/end.2004.18.723

13. Sur RL, Haleblian GE, Cantor D, et al. Efficacy of intravesical ropivacaine injection on urinary symptoms following ureteral stenting: A randomized, controlled study. J Endourol 2008;22:473-8. https://doi.org/10.1089/end.2007.9847

14. Miyaoka R, Monga M. Ureteral stent discomfort: Etiology and management. Indian J Urol 2009;25:45560. https://doi.org/10.4103/0970-1591.57910

15. Damiano R, Autorino R, De Sio $M$, et al. Effect of tamsulosin in preventing ureteral stent- related morbidity: A prospective study. J Endourol 2008;22:651-5. https://doi.org/10.1089/end.2007.0257

16. Joshi HB, Newns N, Stainthorpe A, et al. Ureteral stent symptom questionnaire: Development and validation of a multidimensional quality of life measure. J Urol 2003;169:1060-4. https://doi.org/10.1097/01. ju.0000049198.53424.1d

Correspondence: Dr. Darren Beiko, Department of Urology, Queen's University, Kingston, ON, Canada; beikod@KGH.KARI.NET



Supplementary Fig. 1. Consort diagram. OR: operating room; URS: ureteroscopy; UTI: urinary tract infection. 